\title{
Prognostic value of the Simpson grading scale in modern meningioma surgery: Barrow Neurological Institute experience
}

\author{
Colin J. Przybylowski, MD, Benjamin K. Hendricks, MD, Fabio A. Frisoli, MD, Xiaochun Zhao, MD, \\ Claudio Cavallo, MD, Leandro Borba Moreira, MD, Sirin Gandhi, MD, Nader Sanai, MD, \\ Kaith K. Almefty, MD, Michael T. Lawton, MD, and Andrew S. Little, MD
}

Department of Neurosurgery, Barrow Neurological Institute, St. Joseph's Hospital and Medical Center, Phoenix, Arizona

\begin{abstract}
OBJECTIVE Recently, the prognostic value of the Simpson resection grading scale has been called into question for modern meningioma surgery. In this study, the authors analyzed the relationship between Simpson resection grade and meningioma recurrence in their institutional experience.

METHODS This study is a retrospective review of all patients who underwent resection of a WHO grade I intracranial meningioma at the authors' institution from 2007 to 2017. Binary logistic regression analysis was used to assess for predictors of Simpson grade IV resection and postoperative neurological morbidity. Cox multivariate analysis was used to assess for predictors of tumor recurrence. Kaplan-Meier analysis and log-rank tests were used to assess and compare recurrence-free survival (RFS) of Simpson resection grades, respectively.
\end{abstract}

RESULTS A total of 492 patients with evaluable data were included for analysis, including 394 women (80.1\%) and 98 men (19.9\%) with a mean (SD) age of $58.7(12.8)$ years. The tumors were most commonly located at the skull base $(\mathrm{n}=$ $302 ; 61.4 \%)$ or the convexity/parasagittal region $(n=139 ; 28.3 \%)$. The median (IQR) tumor volume was $6.8(14.3) \mathrm{cm}^{3}$. Simpson grade I, II, III, or IV resection was achieved in 105 (21.3\%), $155(31.5 \%), 52$ (10.6\%), and $180(36.6 \%)$ patients, respectively. Sixty-three of 180 patients (35.0\%) with Simpson grade IV resection were treated with adjuvant radiosurgery. In the multivariate analysis, increasing largest tumor dimension $(p<0.01)$ and sinus invasion $(p<0.01)$ predicted Simpson grade IV resection, whereas skull base location predicted neurological morbidity $(p=0.02)$. Tumor recurrence occurred in 63 patients (12.8\%) at a median (IQR) of $36(40.3)$ months from surgery. Simpson grade I resection resulted in superior RFS compared with Simpson grade II resection $(p=0.02)$, Simpson grade III resection $(p=0.01)$, and Simpson grade IV resection with adjuvant radiosurgery $(p=0.01)$ or without adjuvant radiosurgery $(p<0.01)$. In the multivariate analysis, Simpson grade I resection was independently associated with no tumor recurrence $(p=0.04)$. Simpson grade II and III resections resulted in superior RFS compared with Simpson grade IV resection without adjuvant radiosurgery $(p<0.01)$ but similar RFS compared with Simpson grade IV resection with adjuvant radiosurgery $(p=0.82)$. Simpson grade IV resection with adjuvant radiosurgery resulted in superior RFS compared with Simpson grade IV resection without adjuvant radiosurgery $(p<0.01)$.

CONCLUSIONS The Simpson resection grading scale continues to hold substantial prognostic value in the modern neurosurgical era. When feasible, Simpson grade I resection should remain the goal of intracranial meningioma surgery. Simpson grade IV resection with adjuvant radiosurgery resulted in similar RFS compared with Simpson grade II and III resections.

https://thejns.org/doi/abs/10.3171/2020.6.JNS20374

KEYWORDS meningioma; prognosis; recurrence; Simpson grading scale; oncology

I N 1957, Simpson's landmark publication demonstrated a stepwise decrease in meningioma recurrence with increasingly aggressive resection, namely, coagulation and resection of the tumor's dural attachment. ${ }^{1}$ Maximal safe tumor and dural removal was thus defined as the gold standard neurosurgical management of these tumors for several decades. ${ }^{2-5}$ Since 2010 , several publications have questioned the relevance of Simpson's grading scale in modern meningioma surgery. ${ }^{6-9}$ In these studies, meningioma recurrence rates did not differ among Simpson resec-

ABBREVIATIONS $m R S=$ modified Rankin Scale; RFS = recurrence-free survival.

SUBMITTED February 6, 2020. ACCEPTED June 3, 2020.

INCLUDE WHEN CITING Published online October 23, 2020; DOI: 10.3171/2020.6.JNS20374. 
tion grades I, II, and III. Because these studies reviewed patient outcomes from only the recent neurosurgical era, however, their follow-up times were limited, which may have underpowered their statistical analyses. Nonetheless, changes in the WHO classification criteria of meningiomas and advances in neurosurgical technologies, such as preoperative embolization techniques, neuronavigation software, and high-quality MRI, were hypothesized to explain their findings. ${ }^{6-8}$

Our institutional approach to intracranial meningioma surgery is to attempt maximal safe resection with the goal of Simpson grade I resection where feasible. We also employ adjuvant radiosurgery after Simpson grade IV resection based on individualized clinical context and patient preference. In this study, we explored the prognostic value of the Simpson grading scale by analyzing our comprehensive institutional experience. Unlike previous studies on this topic, our analysis includes the use of adjuvant radiosurgery after subtotal resection to provide a more practical display of modern clinical management.

\section{Methods \\ Patient Selection}

This study is a retrospective review of all patients who underwent resection of a WHO grade I intracranial meningioma at our institution between January 1, 2007, and December 31, 2017. The 2007 WHO guidelines were used by the neuropathology department from 2007 to 2015, and 2016 guidelines were used from 2016 to $2017 . .^{10}$ The exclusion criteria included atypical or anaplastic histopathology, age $<18$ years, a diagnosis of meningiomatosis or neurofibromatosis, and follow-up time $<6$ months. For asymptomatic patients, resection was offered at the discretion of the attending neurosurgeon. However, it was typically offered to patients with large or growing meningiomas. Informed consent for surgery was obtained from all patients or their guardians before the procedures described in this study were performed. Informed consent for the study analysis was not required due to the retrospective nature of the study. This study was approved by the institutional review board of St. Joseph's Hospital and Medical Center in Phoenix, Arizona.

\section{Surgical Strategy}

The surgical strategy for intracranial meningioma resection has been discussed extensively. ${ }^{2}$ We follow the tenets classically described to maximize tumor resection, including 1) adequate bony removal for complete tumor exposure, 2) early tumor devascularization along the dural attachment, 3) tumor debulking for ease of surgical manipulation, 4) dissection of the tumor's arachnoid plane from the brain parenchyma and critical structures, and 5) coagulation and resection of the dural attachment when possible (Fig. 1).

\section{Clinical and Radiographic Assessment}

Direct chart and neuroimaging reviews were performed to obtain patient, tumor, surgical, and outcome data. Preoperative tumor volume was estimated with manual segmentation on contrast-enhanced T1-weighted MRI using the following formula: $x y z / 2$. The major dural sinuses evaluated for tumor invasion (sinus invasion) included the cavernous sinus, sagittal sinus, torcula, transverse sinus, and sigmoid sinus. The major intracranial arteries evaluated for tumor encasement (artery encasement) included the internal cerebral, middle cerebral, vertebral, and basilar arteries. Operative reports and immediate ( $<48$ hours) postoperative MRI were reviewed to determine the Simpson resection grade. ${ }^{1}$ In this study, any residual solid tumor (including tumor confined only to the intrasinus region) was considered Simpson grade IV. Radiographic followup was individualized on the basis of attending neurosurgeon preference. However, follow-up typically occurred at 1 -year intervals after the operation for 3 years and then every 3 years thereafter. Tumor recurrence was defined by radiographic growth (any increase in nodular enhancement not believed to be evolving postoperative changes by the attending neurosurgeon or neuroradiologist) on serial MRI in comparison with the immediate postoperative MRI findings. Patients were typically followed up in the outpatient clinic by the attending neurosurgeon at 4 weeks, 3 months, and 1 year after the operation. Patients with residual tumor or postoperative neurological deficits were often followed up more frequently at the discretion of the attending neurosurgeon. Additionally, adjuvant radiosurgery was offered to patients with residual tumor at the discretion of the attending neurosurgeon and in agreement with individual patient preference. Neurological morbidity was defined by the development of a new or worsening neurological deficit without a complete recovery to baseline at last follow-up. In addition to focal neurological deficits, clinical outcomes were measured using the modified Rankin Scale (mRS). An mRS score $>2$ was considered to indicate poor functional status.

\section{Statistical Methods}

Continuous variables are presented as means (SDs), and categorical variables are presented as frequencies (\%). Univariate and subsequent multivariate binary logistic regression models were constructed to analyze for predictors of Simpson grade IV resection and postoperative neurological morbidity. Univariate and multivariate Cox regression analyses were performed to identify factors associated with tumor recurrence. The variables analyzed included age, sex, preoperative mRS score, tumor laterality, tumor location, largest tumor dimension, tumor volume, sinus invasion, artery encasement, histopathology subtype, and Simpson resection grade. The Bonferroni correction was applied to the $\mathrm{p}$ value for univariate significance to reduce familywise error. Kaplan-Meier analysis and log-rank tests were used to assess and compare recurrence-free survival (RFS) of Simpson resection grades, respectively. IBM SPSS software (version 25, IBM Corp.) was used for all analyses; $\mathrm{p}<0.05$ was considered statistically significant.

\section{Results}

A total of 1435 patients underwent resection of a WHO grade I intracranial meningioma at our institution during the study period. Of these, 689 patients (48.0\%) were 
A
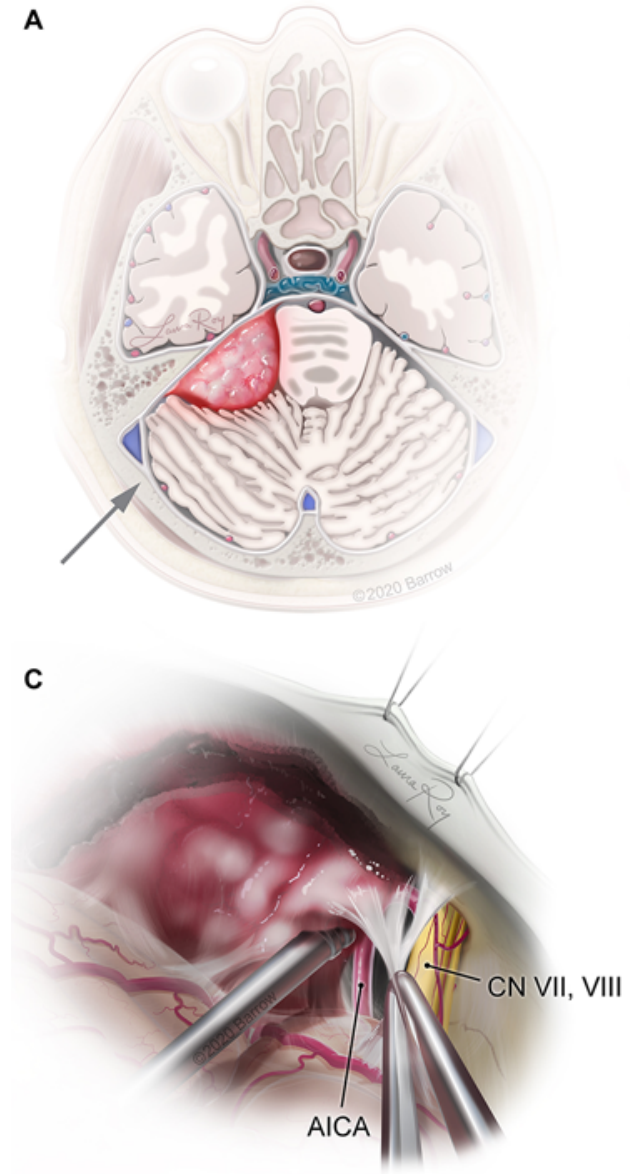

B

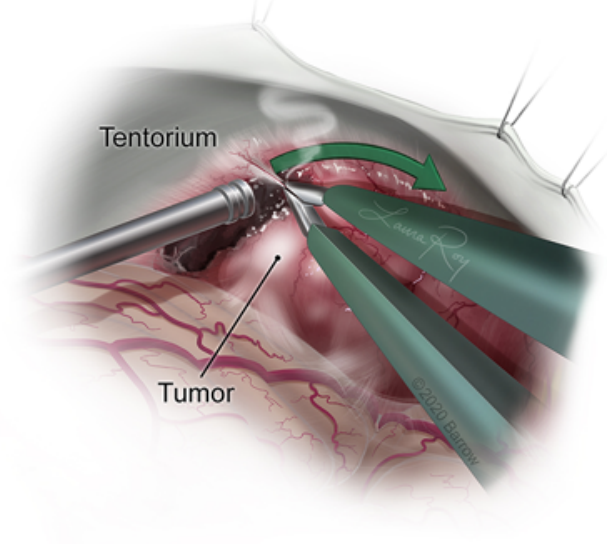

D

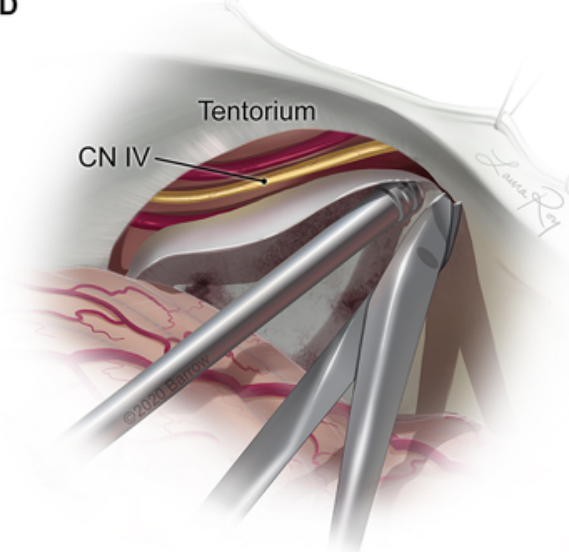

FIG. 1. A: Representative illustration of a Simpson grade I resection of a right-sided tentorial meningioma approached via a retrosigmoid craniotomy (arrow). B: The tumor is first devascularized along its dural attachment. C: The tumor arachnoid plane is preserved and carefully dissected to protect the brain parenchyma, cranial nerves (CNs), and microvasculature. D: After tumor resection, the tentorial attachment is coagulated, cut, and resected, with careful preservation of the traversing CN IV. AICA = anterior inferior cerebellar artery. Used with permission from Barrow Neurological Institute, Phoenix, Arizona. Figure is available in color online only.

excluded because the Simpson grade could not be determined, which was due to a lack of radiographic follow-up or missing operative reports. Two hundred fifty-four patients $(17.7 \%)$ were excluded for lack of clinical follow-up. These exclusions yielded 492 patients (34.3\%) with evaluable data for study inclusion.

\section{Patient and Tumor Characteristics}

The study cohort included 394 women (80.1\%) and 98 men $(19.9 \%)$ with a mean (SD) age of $58.7(12.8)$ years (Table 1). The most common presenting symptoms were cranial neuropathy $(\mathrm{n}=122 ; 24.8 \%)$, headache $(\mathrm{n}=94$; $19.1 \%)$, imbalance and/or dizziness $(n=80 ; 16.3 \%)$, visual disturbance $(n=41 ; 8.3 \%)$, and seizure $(n=40 ; 8.1 \%)$. Eighty-nine patients (18.1\%) were asymptomatic, and 17 patients $(3.5 \%)$ had a poor preoperative mRS score.

Tumor laterality included the left side in 213 patients (43.3\%), right side in 204 patients (41.5\%), and midline in 75 patients $(15.2 \%)$. The tumors were most commonly located at the skull base $(\mathrm{n}=302 ; 61.4 \%)$ or the convexity/ parasagittal region $(\mathrm{n}=139 ; 28.3 \%)$, and the median (IQR) tumor volume was $6.8(14.3) \mathrm{cm}^{3}$. Ninety-six tumors $(19.5 \%)$ invaded into a major dural sinus, and 49 tumors $(10.0 \%)$ encased a major intracranial artery. The most common histopathological subtypes were transitional $(\mathrm{n}=$ $183 ; 37.2 \%)$, fibrous $(\mathrm{n}=101 ; 20.5 \%)$, and meningothelial $(\mathrm{n}=99 ; 20.1 \%)$.

\section{Simpson Grade}

Simpson grade I, II, III, or IV resection was achieved in 105 (21.3\%), 155 (31.5\%), 52 (10.6\%), and 180 (36.6\%) patients, respectively. In the univariate analysis, increasing preoperative $\mathrm{mRS}$ score $(\mathrm{p}<0.01)$, increasing largest tumor dimension $(\mathrm{cm})(\mathrm{p}<0.01)$, sinus invasion $(\mathrm{p}<0.01)$, artery encasement $(\mathrm{p}<0.01)$, and nonconvexity location $(\mathrm{p}$ $<0.01$ ) were associated with Simpson grade IV resection. The multivariate logistic regression model showed that increasing largest tumor dimension $(\mathrm{p}<0.01$; OR $1.4[95 \%$ CI 1.2-1.6]) and sinus invasion ( $<<0.01$; OR 11.9 [95\% CI 6.4-22.3]) independently predicted Simpson grade IV 
TABLE 1. Demographic and clinical characteristics of 492 patients who underwent resection of a WHO grade I intracranial meningioma

\begin{tabular}{|c|c|}
\hline Characteristic & Value \\
\hline Age in yrs, mean (SD) & $58.7(12.8)$ \\
\hline Female sex & $394(80.1)$ \\
\hline \multicolumn{2}{|l|}{ Preoperative mRS score } \\
\hline 0 & $67(13.6)$ \\
\hline 1 & $279(56.7)$ \\
\hline 2 & $129(26.2)$ \\
\hline 3 & $17(3.5)$ \\
\hline \multicolumn{2}{|l|}{ Tumor laterality } \\
\hline $\mathrm{Lt}$ & $213(43.3)$ \\
\hline Rt & $204(41.5)$ \\
\hline Midline & $75(15.2)$ \\
\hline Max tumor diameter in $\mathrm{cm}$, mean (SD) & $2.9(1.3)$ \\
\hline Tumor vol in $\mathrm{cm}^{3}$, median (IQR) & $6.8(14.3)$ \\
\hline \multicolumn{2}{|l|}{ Tumor location } \\
\hline Skull base & $302(61.4)$ \\
\hline Convexity/parasagittal & $139(28.3)$ \\
\hline Tentorial/pineal & $32(6.5)$ \\
\hline Parafalcine & $9(1.8)$ \\
\hline Cerebellar & $8(1.6)$ \\
\hline Intraventricular & $2(0.4)$ \\
\hline Sinus invasion & $96(19.5)$ \\
\hline Artery encasement* & $49(10.0)$ \\
\hline \multicolumn{2}{|l|}{ Histopathology } \\
\hline Transitional & $183(37.2)$ \\
\hline Fibrous & $101(20.5)$ \\
\hline Meningothelial & $99(20.1)$ \\
\hline Secretory & $30(6.1)$ \\
\hline Psammomatous & $17(3.5)$ \\
\hline Angiomatous & $12(2.4)$ \\
\hline Microcystic & $6(1.2)$ \\
\hline Unknown & $44(8.9)$ \\
\hline
\end{tabular}

Values are expressed as number of patients (\%) unless otherwise indicated. * Determined by review of preoperative MRI findings.

resection. Sixty-three of 180 patients $(35.0 \%)$ with Simpson grade IV resection were treated with adjuvant radiosurgery, whereas 117 patients $(65.0 \%)$ were not. Of the 63 patients who underwent adjuvant radiosurgery, 41 (65.1\%) had residual tumors invading a major dural sinus. Table 2 shows the Simpson resection grades of the two major anatomical locations represented in this study: skull base and convexity/parasagittal. Simpson grade I resection was rare among patients with skull base meningiomas (4.0\%) but common among patients with convexity/parasagittal meningiomas $(61.2 \%)$.

\section{Morbidity}

The incidence of neurological morbidity following resection was $7.9 \%(n=39)$. Permanent neurological deficits
TABLE 2. Sinus invasion and Simpson grade among 441 patients with skull base and convexity/parasagittal meningiomas

\begin{tabular}{ccc}
\hline & \multicolumn{2}{c}{ No. of Patients (\%) } \\
\cline { 2 - 3 } Variable & $\begin{array}{c}\text { Skull Base } \\
(\mathrm{n}=302)\end{array}$ & $\begin{array}{c}\text { Convexity/Parasagittal } \\
(\mathrm{n}=139)\end{array}$ \\
\hline Sinus invasion & $63(20.9)$ & $13(9.4)$ \\
\hline Simpson grade & & \\
\hline I & $12(4.0)$ & $85(61.2)$ \\
\hline II & $126(41.7)$ & $16(11.5)$ \\
\hline III & $46(15.2)$ & $4(2.9)$ \\
\hline IV & $118(39.1)$ & $34(24.5)$ \\
\hline
\end{tabular}

included cranial neuropathy $(\mathrm{n}=20 ; 4.1 \%)$, visual decline $(n=5 ; 1.0 \%)$, cognitive decline $(n=5 ; 1.0 \%)$, hemiparesis $(\mathrm{n}=4 ; 0.8 \%)$, sensory deficit $(\mathrm{n}=4 ; 0.8 \%)$, and aphasia $(\mathrm{n}=1 ; 0.2 \%)$. In the univariate analysis, Simpson grade I resection $(\mathrm{p}<0.01)$ and convexity/parasagittal location ( $p<0.01$ ) were negative predictors of neurological morbidity, whereas skull base location was a positive predictor of neurological morbidity $(\mathrm{p}<0.01)$. In the multivariate analysis, only skull base location remained significant as a predictor of postoperative neurological morbidity $(\mathrm{p}=$ 0.02; OR 2.4 [95\% CI 1.1-5.2]). Other surgical complications included hydrocephalus $(\mathrm{n}=18 ; 3.7 \%)$, hemorrhage and/or stroke ( $\mathrm{n}=16 ; 3.3 \%)$, infection and/or wound breakdown $(\mathrm{n}=16 ; 3.3 \%)$, and cerebrospinal fluid leak $(\mathrm{n}=14$; $2.8 \%$ ). Postoperative medical complications included diabetes insipidus $(\mathrm{n}=7 ; 1.4 \%)$, syndrome of inappropriate antidiuretic hormone $(n=4 ; 0.8 \%)$, respiratory failure $(n=$ $4 ; 0.8 \%)$, urinary tract infection $(n=4 ; 0.8 \%)$, pneumonia $(\mathrm{n}=3 ; 0.6 \%)$, deep venous thrombosis $(\mathrm{n}=2 ; 0.4 \%)$, pulmonary embolism $(\mathrm{n}=2 ; 0.4 \%)$, and myocardial infarction $(\mathrm{n}=1 ; 0.2 \%)$. The median (IQR) estimated blood loss in surgery was $250(250) \mathrm{mL}$, but transfusion rates could not be accurately calculated. No patients experienced new or worsening permanent neurological deficits following postoperative radiosurgery.

\section{Tumor Recurrence and Simpson Resection Grade}

Tumors recurred in 63 patients $(12.8 \%)$ at a median (IQR) of 36 (40.3) months after surgery. Of these 63 patients, $9(14.3 \%)$ were newly symptomatic at the time of recurrence. Thus, the overall rate of symptomatic recurrence in this series was 1.8\% (9 of 492 patients). The 1-, 3-, and 5 -year actuarial RFS rates for the entire study cohort were $98.6 \%, 90.2 \%$, and $81.6 \%$, respectively (Fig. 2A).

Simpson resection grade was highly predictive of RFS, both in the entire cohort ( $p<0.01$; Fig. $2 B$ ) and in the subgroup analysis of patients with skull base meningiomas ( $p<0.01$; Fig. 2C). The 5-year actuarial RFS rates for Simpson grades I, II, III, IV without radiosurgery, and IV with radiosurgery were $94.6 \%, 88.3 \%, 85.1 \%, 55.6 \%$, and $85.0 \%$, respectively. Direct comparisons among different Simpson resection grades are shown in Fig. 3. Simpson grade I resection resulted in superior RFS compared with Simpson grade II resection ( $\mathrm{p}=0.02$; Fig. 3A), Simpson grade III resection ( $\mathrm{p}=0.01$; Fig. $3 \mathrm{~B})$, and Simpson grade 

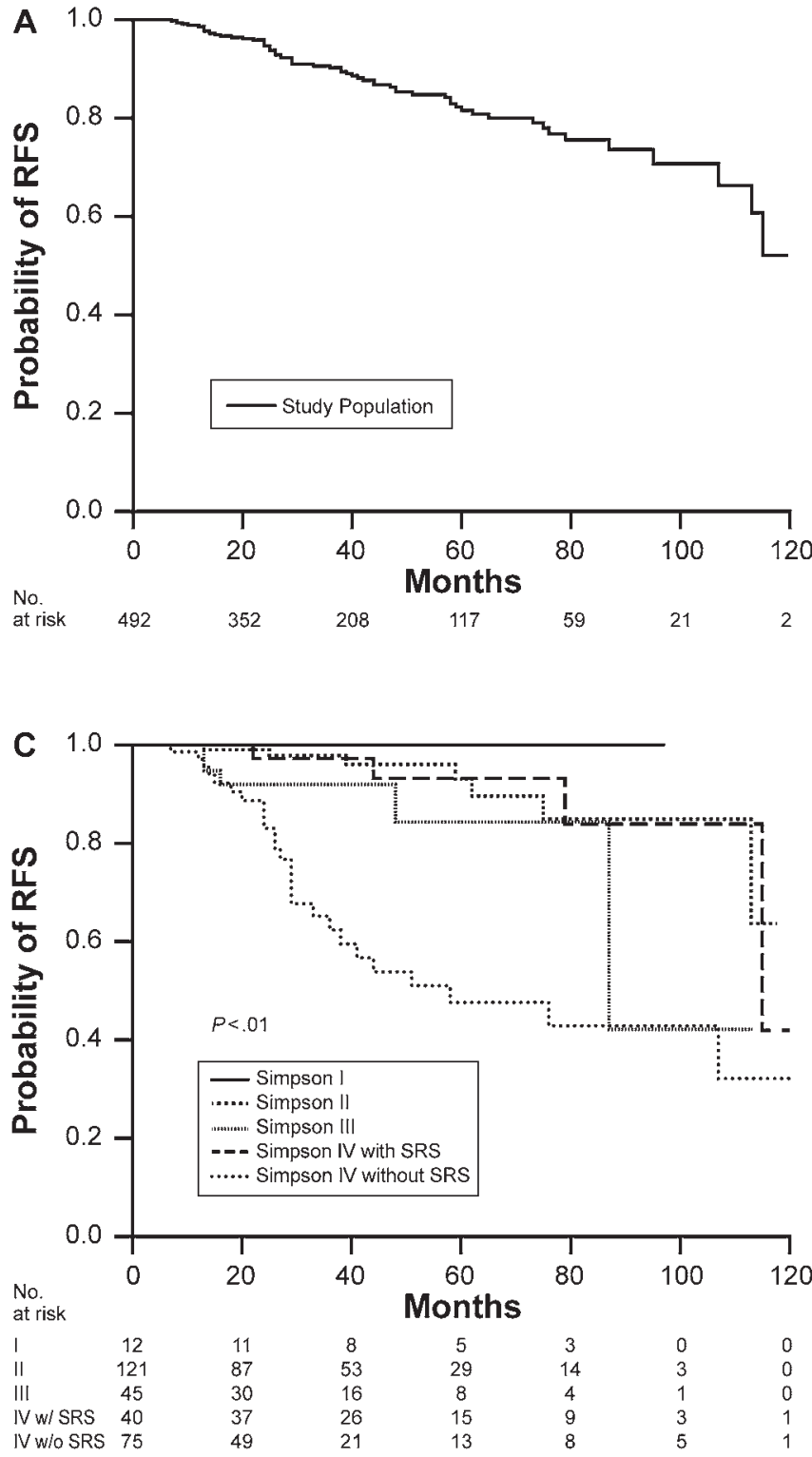

IV resection with adjuvant radiosurgery $(\mathrm{p}=0.01$; Fig. $3 \mathrm{C})$ or without adjuvant radiosurgery $(\mathrm{p}<0.01)$. Simpson grade II and III resections resulted in superior RFS compared with Simpson grade IV resection without adjuvant radiosurgery ( $p<0.01$; Fig. 3D) but similar RFS compared with Simpson grade IV resection with adjuvant radiosurgery $(p=0.82)$. Simpson grade IV resection with adjuvant radiosurgery resulted in superior RFS compared with Simpson grade IV resection without adjuvant radiosurgery $(\mathrm{p}<0.01)$. The Cox multivariate analysis showed that Simpson grade I resection was independently associated with no tumor recurrence ( $\mathrm{p}=0.04$; OR 0.21 [95\% CI 0.05-0.90]), whereas Simpson grade IV resection without radiosurgery $(\mathrm{p}<0.01$; OR 3.2 [95\% CI 1.9-5.3]) and artery encasement ( $\mathrm{p}=0.02$; OR 2.1 [95\% CI 1.1-3.8]) were independently associated with tumor recurrence. Patients with recurrence $(\mathrm{n}=63)$ were treated with radiosurgery alone $(\mathrm{n}=31 ; 49.2 \%)$, resection alone $(\mathrm{n}=18 ; 28.6 \%)$, con-

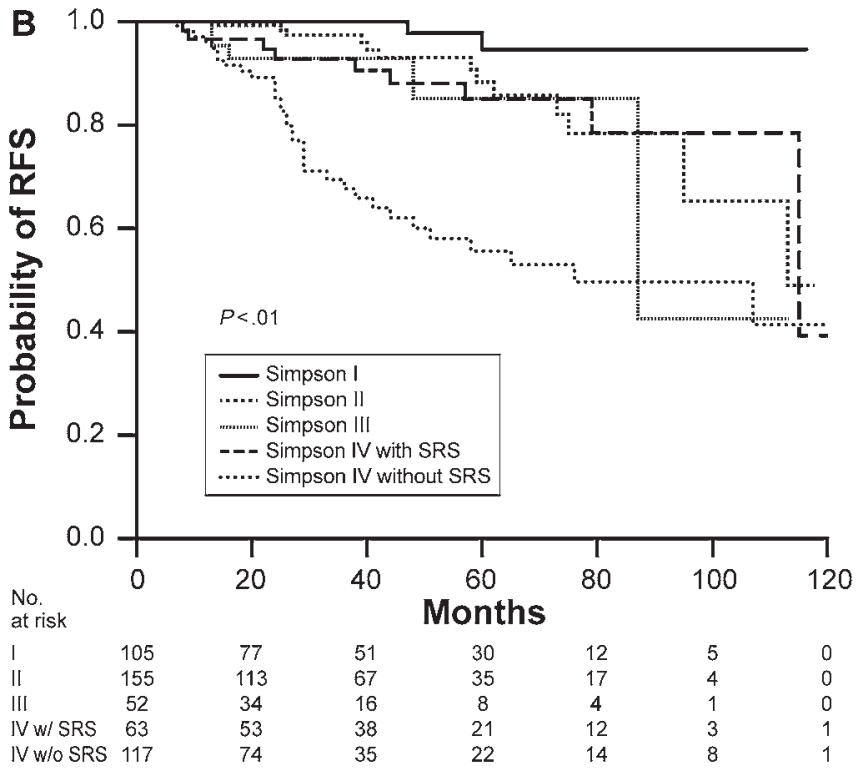

FIG. 2. Kaplan-Meier RFS curves of the entire study cohort (A), the entire study cohort stratified by Simpson resection grade and postoperative stereotactic radiosurgery (B), and the subgroup of patients with skull base meningiomas stratified by Simpson resection grade (C). SRS = stereotactic radiosurgery.

servative management $(\mathrm{n}=11 ; 17.5 \%)$, or resection plus radiosurgery $(\mathrm{n}=3 ; 4.8 \%)$. However, outcomes following recurrence were not analyzed for this study.

\section{Final Clinical Outcome}

The mean (SD) follow-up duration for the entire cohort was 44.8 (30.5) months. The mean (SD) follow-up times for patients with Simpson grade I-III and Simpson grade IV resections were 42.9 (28.7) and 48.2 (33.2) months, respectively $(\mathrm{p}=0.08)$. The outcome of the major presenting symptom was determined in 485 patients. The major presenting symptom improved in 237 patients (48.9\%), remained stable in 181 patients $(37.3 \%)$, and worsened in 67 patients $(13.8 \%)$. The final $\mathrm{mRS}$ score was able to be determined in 490 patients. At last follow-up, 465 patients (94.9\%) were functionally independent (mRS 0-2), and 25 patients (5.1\%) were not. 

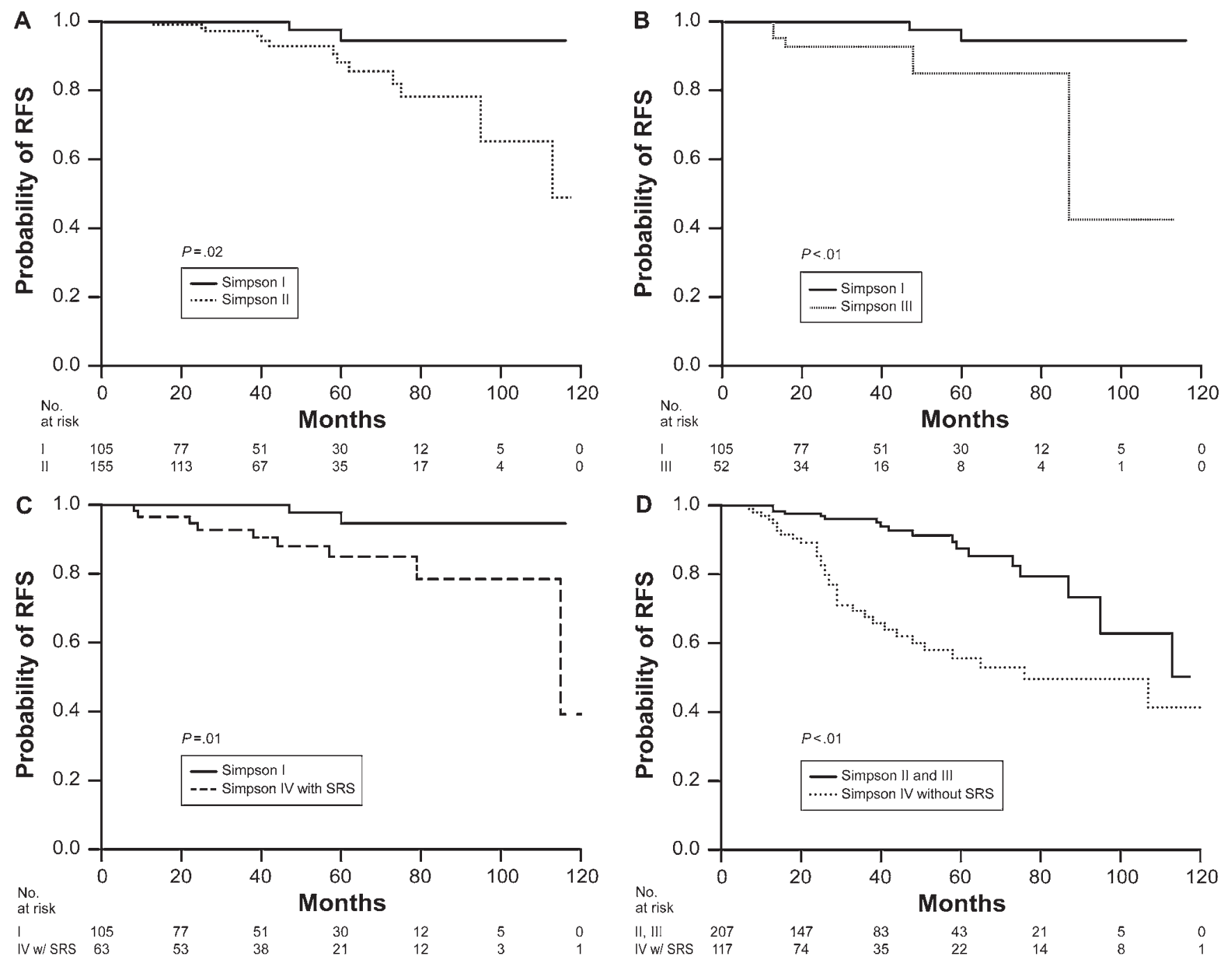

FIG. 3. Kaplan-Meier RFS curves comparing Simpson grade I resection versus Simpson grade II resection (A), Simpson grade I resection versus Simpson grade III resection (B), Simpson grade I resection versus Simpson grade IV resection with adjuvant stereotactic radiosurgery (C), and Simpson grade II and III resections versus Simpson grade IV resection without SRS (D). The log-rank test was used for the significance of the comparison.

\section{Discussion}

We performed a retrospective review of our 10-year experience with WHO grade I intracranial meningioma resection to study the association between Simpson resection grade and meningioma recurrence. We found that the Simpson grading scale was highly predictive of meningioma recurrence, with Simpson grade I resection offering superior tumor control to both Simpson grade II and III resections as well as Simpson grade IV resection combined with adjuvant radiosurgery. Similar to Simpson's original report, we found a stepwise increase in meningioma recurrence with increasing Simpson resection grade (Fig. 2A).

Our finding that the Simpson grading scale maintains its relevance in modern meningioma surgery agrees with two recent publications. Nanda et al. ${ }^{11}$ analyzed the surgical outcomes of 458 patients with WHO grade I meningiomas. Their overall tumor recurrence rates for Simpson grades I, II, III, and IV were $5 \%, 22 \%, 31 \%$, and $35 \%$, respectively. The difference in the median RFS time was statistically significant among Simpson grades $(p=0.001)$. Additionally, Simpson grade I resection was an independent predictor of RFS in the multivariate analysis $(\mathrm{p}=$ 0.02 ), in agreement with our results. Similarly, Winther and Torp ${ }^{12}$ recently analyzed the surgical outcomes of 113 patients with WHO grade I meningiomas and found the 15-year recurrence rates after Simpson resection grades I, II, III, and IV to be $2.9 \%, 12.5 \%, 31.3 \%$, and $64.3 \%$, respectively. In their analysis, a meningioma was 13.1 times more likely to recur following a Simpson grade III resection than following a Simpson grade I resection $(\mathrm{p}=0.02)$.

Unlike previous studies, we divided Simpson grade IV patients into two groups based on whether they received adjuvant radiosurgery. This grouping provided a more accurate representation of patient subgroups to the modern neurosurgeon. Our data demonstrated that Simpson grade 
TABLE 3. Comparison of recent studies evaluating the Simpson grading scale for predicting intracranial meningioma recurrence in different WHO pathological grades

\begin{tabular}{|c|c|c|c|c|}
\hline Authors \& Year & $\begin{array}{c}\text { No. of } \\
\text { Patients }\end{array}$ & $\begin{array}{l}\text { Mean or Median } \\
\text { Follow-Up (mos)* }\end{array}$ & RFS Among Patients w/ Simpson Grades I-III & $\begin{array}{l}\text { Statistically Significant Difference } \\
\text { Among Simpson Grades I-III }\end{array}$ \\
\hline \multicolumn{5}{|l|}{ WHO grade I } \\
\hline Sughrue et al., $2010^{6}$ & 373 & Median 44.4 & 5-yr RFS: $95 \%$ (I) vs $85 \%$ (II) vs $88 \%$ (III) & No \\
\hline Oya et al., $2012^{7}$ & 240 & NR & 5-yr RFS: $97.6 \%$ (I) vs $87.7 \%$ (II) vs $84.1 \%$ (III) & No \\
\hline Heald et al., $2014^{8}$ & 183 & Mean 35.3 & 3-yr RFS: $95 \%$ (I) vs $87 \%$ (II) & No \\
\hline Otero-Rodriguez et al., $2016^{9}$ & 224 & Median 60 & 5-yr RFS: $97 \%$ (I) vs $95 \%$ (II) vs $98 \%$ (III) & No \\
\hline Nanda et al., $2017^{11}$ & 458 & Mean 54 & Overall: $95 \%$ (I) vs $78 \%$ (II) vs $69 \%$ (III) & Yes \\
\hline Winther \& Torp, $2017^{12}$ & 113 & Median 123 & 5-yr RFS: $97.1 \%$ (I) vs $91.3 \%$ (II) vs $86.7 \%$ (III) & Yes \\
\hline Present study & 492 & Mean 44.8 & 5-yr RFS: $94.6 \%$ (I) vs $88.3 \%$ (II) vs $85.1 \%$ (III) & Yes \\
\hline \multicolumn{5}{|l|}{ WHO grades I-III } \\
\hline Ehresman et al., $2018^{16}$ & 572 & NR & 4-yr RFS: $95 \%$ (I) vs $89.2 \%$ (II) vs $87.9 \%$ (III) & No \\
\hline Hasseleid et al., $2012^{17}$ & $391 \dagger$ & Median 85.2 & Overall: $96.8 \%$ (I) vs $84.8 \%$ (II) vs $87.5 \%$ (III) & Yes \\
\hline Gousias et al., $2016^{18}$ & $901 \ddagger$ & Median 62 & 10-yr RFS: $91.5 \%$ (I) vs $81.2 \%$ (II) & Yes \\
\hline
\end{tabular}

NR $=$ not reported.

* Approximated in months for reader comparison.

$\dagger$ Location was confined to the convexity.

$\ddagger$ Included 46 patients (5.1\%) with spinal meningiomas.

IV patients who did not receive adjuvant radiosurgery experienced the highest rate of tumor recurrence among all groups (Fig. 2B). Furthermore, Simpson grade IV resection without adjuvant radiosurgery was an independent predictor of tumor recurrence in the multivariate analysis $(\mathrm{p}<0.01)$. On the contrary, Simpson grade IV resection with radiosurgery resulted in similar RFS compared with Simpson grade II or III resection. This finding is in agreement with the growing evidence that adjuvant radiosurgery following subtotal resection can offer excellent tumor control for WHO grade I meningiomas..$^{13,14}$ Complete resection of a meningioma is not always feasible, particularly for skull base meningiomas with extensive involvement of critical neurovascular structures. These data suggest that, in the era of radiosurgery, attempting a Simpson grade II or III resection is unwarranted if it poses a significant risk of neurological compromise. Nonetheless, we would not advocate for Simpson grade IV resection with radiosurgery when Simpson grade II or III resection is otherwise safe and possible, as the additional radiosurgery procedure would add unnecessary morbidity and cost to patient care.

When considering the outcomes of Simpson grade IV resection with and without radiosurgery, it is important to note that, due to the retrospective nature of this study, unaccounted-for differences in residual tumor characteristics, such as size, location, and invasiveness, may have affected the attending neurosurgeon's decision to recommend adjuvant radiosurgery. It is further important to note that the primary outcome of this study was first-time radiographic recurrence, which has limitations in translation to clinical practice. After Simpson grade IV resection, it is often reasonable to follow up residual tumors conservatively with serial imaging and offer "salvage" radiosurgery only at the first sign of tumor recurrence. Considering the low rate of symptomatic recurrence in this study (1.8\%), delaying radiosurgery until there is recurrence does not appear to be associated with significant clinical decline. This wait-and-see approach may also provide more costeffective neurosurgical care. However, on the basis of the results of this study, we question whether "upfront" radiosurgery results in improved long-term tumor control compared with salvage radiosurgery. Although this question is clinically important, our study was not designed to answer it. In the absence of a randomized, prospective study, it is difficult to make accurate comparisons between these two groups of patients (upfront vs salvage radiosurgery) because a neurosurgeon's decision to offer upfront radiosurgery is likely based on a myriad of factors that are difficult to control retrospectively, such as subjective judgment and anecdotal experience. Nonetheless, this issue should be an area of future investigation.

Recent questions about the relevance of the Simpson grading scale in modern meningioma surgery have stimulated debate.$^{6-9}$ Most notably, in 2010, Sughrue et al. ${ }^{6}$ found no association between meningioma recurrence and Simpson grade among 373 patients with WHO grade I meningiomas. Notably, this study reported no recurrences in 33 patients with more than 8 years of follow-up, including 15 patients with Simpson grade IV resection. This finding is remarkable, considering that most studies show gradual tumor recurrence with long-term follow-up, especially following Simpson grade IV resection.7, $7,11,12,15$ Our study's Simpson grade IV resection rate, follow-up period, and 5-year RFS rates (for Simpson grades I-III) are similar to those of Sughrue et al. (Table 3). ${ }^{6-9,11,12,16-18}$ We hypothesize that our study's support of the Simpson grading scale, in opposition to Sughrue et al., is a result of greater study power (due to a larger study population) and the expected observation of gradual recurrence with long-term followup. Considering the slow growth rate of WHO grade I meningiomas and the possibility of long-interval tumor recurrence, patient number and follow-up period are critically 
important variables to assess the effects of neurosurgical intervention on these tumors. ${ }^{15}$ The importance of these factors is further underscored by the results of Nanda et al. ${ }^{11}$ and Winther and Torp ${ }^{12}$ whose large patient population $(\mathrm{n}=458)$ and long follow-up period (median 536 weeks), respectively, also appropriately powered their studies to detect clinically significant differences in meningioma recurrence among Simpson grades. Perhaps the most surprising finding of the study by Sughrue et al. ${ }^{6}$ was that no difference in recurrence was observed between Simpson grades I and IV. Other studies that have questioned the prognostic value of Simpson grades I-III have nevertheless observed a stark difference in recurrence rates between gross-total (Simpson grades I-III) and subtotal (Simpson grade IV) resection. ${ }^{7,8} \mathrm{~A}$ comparison of the recent studies evaluating the prognostic value of the Simpson grading scale for WHO grade I meningiomas is shown in Table 3.

The availability of radiosurgery to treat small- to medium-sized meningiomas, either after subtotal resection or as upfront treatment, has caused some neurosurgeons to plan less-aggressive resection strategies preoperatively, especially for skull base meningiomas. ${ }^{19-21}$ Our multivariate analysis found a skull base location to be the only independent predictor of neurological morbidity, highlighting the known complexity of these lesions. In agreement with Nanda et al., ${ }^{11}$ our subgroup analysis of patients with skull base meningiomas demonstrated the continued prognostic value of the Simpson grading scale $(p<0.01$; Fig. 2 C). As others have noted, we found Simpson grade I resection to be rare for skull base meningiomas $(4.0 \%)$ in comparison with convexity/parasagittal meningiomas $(61.2 \%) .{ }^{17,22,23}$ This difference would have most likely been even larger if convexity meningiomas were analyzed alone, as proximity to and invasion of the superior sagittal sinus can preclude Simpson grade I resection of parasagittal tumors. ${ }^{24,25} \mathrm{In}$ a series of 391 convexity meningiomas, Hasseleid et al. ${ }^{17}$ achieved Simpson grade I resection in 315 (81\%) patients. During their observation period of WHO grade I patients, the retreatment rate following a Simpson grade II or III resection was 6.4 times greater than that following a Simpson grade I resection. Alvernia et al. ${ }^{23}$ studied 100 patients with convexity meningiomas and achieved Simpson grade I and III resections in 91 and 9 patients, respectively. Their analysis showed that pial and vascular invasion were associated with meningioma recurrence. In our clinical experience, these factors are also the main reasons that Simpson grade I resection is not achieved for true convexity tumors.

We agree with the notion that overly aggressive attempts at resection to achieve a higher Simpson grade are not indicated at the expense of neurological morbidity. This notion is reflected in our relatively high rate of Simpson grade IV resection over the study period (36.6\%), which may be due to our inclusion of meningiomas with sinus invasion. We rarely follow tumors into the major dural sinuses and perform sinus reconstruction. Some groups have reported success with this aggressive approach, especially for WHO grade II and III meningiomas, ${ }^{26,27}$ but most employ this technique in select situations only, considering the risks of increased intracranial pressure and venous infarction from postoperative sinus occlusion. ${ }^{28,29}$

The inclusion of only WHO grade I meningiomas in this study may limit the generalizability of our results to preoperative surgical planning because the WHO grade is unknown before initial resection. Although the inclusion of all WHO grades into a single study introduces tumor biology as a confounding variable, the association between Simpson resection grade and meningioma recurrence has also been argued in such studies (Table 3). ${ }^{16-18}$ In the largest such series $(\mathrm{n}=901)$, Gousias et al. ${ }^{18}$ found that, in addition to tumor biology (WHO grade and MIB-1 labeling index), meningioma recurrence was directly related to increasing Simpson resection grade $(\mathrm{p}<0.01)$, and Simpson grade II resection compared with grade I resection doubled the 10 -year RFS rate (18.8\% vs $8.5 \%$ ). Because molecular profiling data were not included in our analysis, differences in molecular genetics could have confounded tumor recurrence rates among Simpson resection grades. Recent molecular profiling and epigenetics data suggest that tumor genetics, regardless of the histologically based WHO grade, predict meningioma recurrence. For example, the presence of the TERT promoter mutation, which maintains telomere length of proliferating neoplastic cells, was superior to WHO grade in predicting time to tumor progression among 252 meningioma samples. ${ }^{30}$ Additionally, a DNA methylation-based meningioma classification scheme was superior to the WHO classification in predicting tumor recurrence in 497 meningioma samples. ${ }^{31}$

The single-institution, retrospective nature of our study also exposed it to the referral and patient selection biases of our institution and neurosurgeons. A significant number of patients treated over the study period were lost to follow-up, resulting in only $34.3 \%$ of patients with evaluable data for study inclusion. The Simpson grades of the excluded patients were mostly unknown, which may have biased our outcomes. Nonetheless, the high clinical volume at our center allowed us to effectively study the relationship between Simpson resection grade and WHO grade I meningioma recurrence in one of the largest known series of its type in the literature.

\section{Conclusions}

In this large, single-institution experience of WHO grade I intracranial meningioma resection, there was a strong correlation between Simpson resection grade and meningioma recurrence. Simpson grade I resection resulted in superior RFS compared with Simpson grade II resection, Simpson grade III resection, and Simpson grade IV resection with adjuvant radiosurgery and, when safe, should remain the goal of intracranial meningioma surgery. The Simpson resection grading scale continues to hold substantial prognostic value in the current neurosurgical era, despite suggestions to the contrary.

\section{Acknowledgments}

We thank the staff of Neuroscience Publications at Barrow Neurological Institute for assistance with manuscript preparation.

\section{References}

1. Simpson D. The recurrence of intracranial meningiomas after surgical treatment. J Neurol Neurosurg Psychiatry. 1957; 20(1):22-39. 
2. Al-Mefty O. Operative Atlas of Meningiomas. LippincottRaven; 1998.

3. Jääskeläinen J. Seemingly complete removal of histologically benign intracranial meningioma: late recurrence rate and factors predicting recurrence in 657 patients. A multivariate analysis. Surg Neurol. 1986;26(5):461-469.

4. Stafford SL, Perry A, Suman VJ, et al. Primarily resected meningiomas: outcome and prognostic factors in 581 Mayo Clinic patients, 1978 through 1988. Mayo Clin Proc. 1998; 73(10):936-942.

5. Adegbite AB, Khan MI, Paine KW, Tan LK. The recurrence of intracranial meningiomas after surgical treatment. $J$ Neurosurg. 1983;58(1):51-56.

6. Sughrue ME, Kane AJ, Shangari G, et al. The relevance of Simpson Grade I and II resection in modern neurosurgical treatment of World Health Organization Grade I meningiomas. J Neurosurg. 2010;113(5):1029-1035.

7. Oya S, Kawai K, Nakatomi H, Saito N. Significance of Simpson grading system in modern meningioma surgery: integration of the grade with MIB-1 labeling index as a key to predict the recurrence of WHO Grade I meningiomas. $J$ Neurosurg. 2012;117(1):121-128.

8. Heald JB, Carroll TA, Mair RJ. Simpson grade: an opportunity to reassess the need for complete resection of meningiomas. Acta Neurochir (Wien). 2014;156(2):383-388.

9. Otero-Rodriguez A, Tabernero MD, Munoz-Martin MC, et al. Re-evaluating Simpson grade I, II, and III resections in neurosurgical treatment of World Health Organization grade I meningiomas. World Neurosurg. 2016;96:483-488.

10. Louis DN, Perry A, Reifenberger G, et al. The 2016 World Health Organization classification of tumors of the central nervous system: a summary. Acta Neuropathol. 2016;131(6): 803-820.

11. Nanda A, Bir SC, Maiti TK, et al. Relevance of Simpson grading system and recurrence-free survival after surgery for World Health Organization Grade I meningioma. J Neurosurg. 2017;126(1):201-211.

12. Winther TL, Torp SH. Significance of the extent of resection in modern neurosurgical practice of World Health Organization grade I meningiomas. World Neurosurg. 2017;99:104-110.

13. Przybylowski CJ, Raper DM, Starke RM, et al. Stereotactic radiosurgery of meningiomas following resection: predictors of progression. J Clin Neurosci. 2015;22(1):161-165.

14. Kondziolka D, Patel AD, Kano H, et al. Long-term outcomes after gamma knife radiosurgery for meningiomas. Am J Clin Oncol. 2016;39(5):453-457.

15. Couldwell WT, Cole CD, Al-Mefty O. Patterns of skull base meningioma progression after failed radiosurgery. J Neurosurg. 2007;106(1):30-35.

16. Ehresman JS, Garzon-Muvdi T, Rogers D, et al. The relevance of Simpson grade resections in modern neurosurgical treatment of World Health Organization grade I, II, and III meningiomas. World Neurosurg. 2018;109:e588-e593.

17. Hasseleid BF, Meling TR, Rønning P, et al. Surgery for convexity meningioma: Simpson Grade I resection as the goal: clinical article. J Neurosurg. 2012;117(6):999-1006.

18. Gousias K, Schramm J, Simon M. The Simpson grading revisited: aggressive surgery and its place in modern meningioma management. J Neurosurg. 2016;125(3):551-560.

19. Pollock BE, Stafford SL, Utter A, et al. Stereotactic radiosurgery provides equivalent tumor control to Simpson Grade 1 resection for patients with small- to medium-size meningiomas. Int J Radiat Oncol Biol Phys. 2003;55(4):1000-1005.

20. Cohen-Inbar O, Lee CC, Schlesinger D, et al. Long-term results of stereotactic radiosurgery for skull base meningiomas. Neurosurgery. 2016;79(1):58-68.

21. Kreil W, Luggin J, Fuchs I, et al. Long term experience of gamma knife radiosurgery for benign skull base meningiomas. J Neurol Neurosurg Psychiatry. 2005;76(10):1425-1430.
22. Morokoff AP, Zauberman J, Black PM. Surgery for convexity meningiomas. Neurosurgery. 2008;63(3):427-434.

23. Alvernia JE, Dang ND, Sindou MP. Convexity meningiomas: study of recurrence factors with special emphasis on the cleavage plane in a series of 100 consecutive patients. $J$ Neurosurg. 2011;115(3):491-498.

24. Magill ST, Theodosopoulos PV, McDermott MW. Resection of falx and parasagittal meningioma: complication avoidance. J Neurooncol. 2016;130(2):253-262.

25. Sughrue ME, Rutkowski MJ, Shangari G, et al. Results with judicious modern neurosurgical management of parasagittal and falcine meningiomas. Clinical article. J Neurosurg. 2011; 114(3):731-737.

26. Mantovani A, Di Maio S, Ferreira MJ, Sekhar LN. Management of meningiomas invading the major dural venous sinuses: operative technique, results, and potential benefit for higher grade tumors. World Neurosurg. 2014;82(3-4):455-467.

27. Zeeshan Q, Patel A, Cheng CY, et al. Resection of meningiomas involving major dural venous sinuses: classification, technique, and long-term results. World Neurosurg. 2019;125: e521-e536.

28. Mazur MD, Cutler A, Couldwell WT, Taussky P. Management of meningiomas involving the transverse or sigmoid sinus. Neurosurg Focus. 2013;35(6):E9.

29. Han MS, Kim YJ, Moon KS, et al. Lessons from surgical outcome for intracranial meningioma involving major venous sinus. Medicine (Baltimore). 2016;95(35):e4705.

30. Sahm F, Schrimpf D, Olar A, et al. TERT promoter mutations and risk of recurrence in meningioma. J Natl Cancer Inst. 2015;108(5):djv377.

31. Sahm F, Schrimpf D, Stichel D, et al. DNA methylation-based classification and grading system for meningioma: a multicentre, retrospective analysis. Lancet Oncol. 2017;18(5):682-694.

\section{Disclosures}

Dr. Little: consultant for Spiway and ownership in Kogent.

\section{Author Contributions}

Conception and design: Little, Przybylowski, Frisoli. Acquisition of data: Zhao, Cavallo, Borba Moreira, Gandhi. Analysis and interpretation of data: Przybylowski, Hendricks, Frisoli. Drafting the article: Przybylowski, Hendricks. Critically revising the article: Little, Sanai, Almefty, Lawton. Reviewed submitted version of manuscript: Przybylowski, Hendricks, Frisoli, Zhao, Cavallo, Borba Moreira, Gandhi, Sanai, Almefty, Lawton. Statistical analysis: Przybylowski, Hendricks. Administrative/technical/material support: Little, Lawton. Study supervision: Little.

\section{Supplemental Information}

\section{Previous Presentations}

Portions of this work were presented in abstract form at the 30th Annual Meeting of the North American Skull Base Society, San Antonio, Texas, February 7-9, 2020.

\section{Correspondence}

Andrew S. Little: c/o Neuroscience Publications, Barrow Neurological Institute, St. Joseph's Hospital and Medical Center, Phoenix, AZ.neuropub@barrowneuro.org. 